\title{
Resonant Approximation for Single-Particle Resonances in the Renormalized RPA-Problem*
}

\author{
M. WEIGEL** \\ Lawrence Radiation Laboratory, University of California, Berkeley, California 94720 \\ (Z. Naturforsch. 25 a, 1562-1567 [1970] ; received 19 August 1970)
}

\begin{abstract}
An approximate version of the renormalized RPA-treatment for scattering of nucleons on a hole nucleus is given, in which the shape resonances are treated according to a method of BALASHOv et al. In order to make the results more transparent we take into account the additional influence of the non resonant part of the single-particle continuum with the help of a separable particle-hole force.
\end{abstract}

\section{Introduction}

In a previous work ${ }^{1}$ we have given the equations for the renormalized RPA-problem with inclusion of the single-particle continuum by extending Migdal's quasi-particle approach ${ }^{2}$ to the continu$u^{3}$. We designed a model in which the so called nuclear-structure problem (only bound singleparticle orbits are included) was used as a zeroorder solution. In this model one could first solve the nuclear-structure problem with the full effective particle-hole force. The influence of the continuum was taken into account by approximating all matrix elements containing continuum single-particle states with a separable force so avoiding the original complicated Fredholm proplem. This is due to the fact that the Fredholm determinant degenerates for a separable force. The details as well as further references can be found in Refs. ${ }^{1}, 3$. The model implied the asumption that the solution for the nuclear-structure problem is already a good approximation for the corresponding "bound-state" solution with inclusion of the single-particle continuum. But in some cases it is well known that one has to include single-particle resonances-for instance the $1 \mathrm{~d}_{3 / 2}$-resonance in 160 -in order to obtain a satisfactory solution for the nuclear-

* Work performed under the auspices of the U.S. Atomic Energy Commision.

** On leave from the Sektion Physik der Universität München, Munich, Germany.

1 M. Weigel, Phys. Rev. (C) 1, 1647 (1970); Lawrence Radiation Laboratory Report UCRL-18994.

2 A. B. Migdal, "Theory of Finite Fermi Systems and Applications to Atomic Nuclei," Interscience Publ., New York 1967.

3 M. Weigel, Nucl. Phys. A 137, 629 [1969].

4 L. Garside and W. M. MacDonald, Phys. Rev. 138, B $582[1965]$. structure problem. We have been able to overcome this difficulty by applying the method of GARSIDE and MacDonald ${ }^{4,5}$. But it turns out that the resulting equations are rather complicated, so one might try an approximate treatment by the use of a "resonant approximation" for the resonant singleparticle states 6,7 . We will give in the second section the relevant definitions and approximations needed in such an attempt. The resulting equations are derived in the third section. In order to make the structure of the problem more transparent a solvable model is presented in the fourth section, in which all matrix elements containing non resonant continuum single-particle states are approximated by a separable force. We will restrict ourselves to the case of one shape resonance only, since the generalization to several channels can be achieved easily by following the same road as in the one channel case.

\section{General RPA-Formalism and the Definition of the Resonant Approximation}

In Ref. ${ }^{1}$ the following equation for the particlehole amplitude was obtained ${ }^{8}$ (A; I.12):

5 L. Garside and M. Weigel, Phys. Rev. (C) 2, 374 (1970); Lawrence Radiation Laboratory Report UCRL-19538.

6 V. V. Balashov et al., Sov. J. Nucl. Phys. (Engl. Transl.) 2, 461 [1966].

7 H. J. Unger, Nucl. Phys. A 104, 564 [1967].

8 In this paper we are using the same notations and definitions as in Ref. ${ }^{1}$. We refer to Ref. ${ }^{1}$ as A, so that (A; I.1) means Eq. (I.1) of Ref. ${ }^{1} . \sum_{\sigma \lambda}$ means summation over the discrete variables as well as integration over the continuum variables. 


$$
\begin{aligned}
\tilde{\varrho}_{v \mu, M}= & \left(n_{v}-n_{\mu}\right) \frac{1}{E_{M}+\varepsilon_{\mu}-\varepsilon_{\nu}-i \eta\left(n_{\nu}-n_{\mu}\right)} \\
& \cdot\left\{\delta_{k_{0} \nu} \delta_{j_{0} \mu}(-i \eta) \delta_{M, S}\right. \\
& \left.-2 \pi \sum_{\sigma \lambda} \tilde{I}_{\nu \sigma \mu \lambda}(\omega) \tilde{\varrho} \lambda \sigma, M\right\} .
\end{aligned}
$$

Here $\tilde{\varrho}_{v \mu, M}$ is the quasi-particle-hole amplitude:

$$
\tilde{\varrho}_{v \mu, M}:=\left(z_{\nu} z_{\mu}\right)^{-1 / 2}\left\langle 0\left|\psi_{\mu}^{\dagger} \psi_{\nu}\right| M\right\rangle,
$$

where $\psi_{\mu}^{\dagger}$ and $\psi_{\mu}$ are the Schroedinger creationand annihilation operators, respectively, of a nucleon with the quantum number set $\mu$ defined by a suitable shell-model hamiltonian. For continuum wavefunctions we choose the standing wave boundary condition. The $z_{v}^{\prime} s$ are Migdal's renormalization constants $^{2} ; 2 \pi \tilde{I}$ is the renormalized particle-hole matrix element. By $|M\rangle$ we denote a scattering state as well as a bound state $|B\rangle$. Since we assume the target nucleus to be a closed shell nucleus plus one quasi-hole, we can specify the scattering state $|S\rangle$ by the quantum number set $k_{0}\left(=\varepsilon_{k_{0}}\right.$, $j_{k_{0}}, l_{k_{0}}, m_{k_{0}}, t_{k_{0}}$ ) of the incoming particle plus the quantum number set $j_{0}$ of the corresponding quasihole. Hence $|S\rangle$ means more explicitely $\left|S ; k_{0}, j_{0}\right\rangle$. With $n_{v}$ we denote the quasiparticle occupation number for the state $v$ with respect to the groundstate $|0\rangle$ of the compound-nucleus. The zeropoint of the energy is chosen to be ground state energy of the compound nucleus.

One knows ${ }^{6,7}$ that within the range of the singleparticle potential the continuum wave function $|p\rangle$ in the resonance channel $\tilde{\xi}$ can be represented in the vicinity of an isolated pol by

$|p\rangle \approx C\left(\varepsilon_{p}\right)|\xi\rangle$ for $\bar{p}=\bar{\xi}, 0 \leq \varepsilon_{p} \leq \varepsilon_{\max }$,

with $\langle\xi \mid \xi\rangle=1$. Here, with $\bar{p}, \bar{\xi}$ etc. we specify the channels-for instance $\bar{p}:=j_{p}, l_{p}, t_{p} . C\left(\varepsilon_{p}\right)$ has the following structure:

$$
C\left(\varepsilon_{p}\right):=\left\{\frac{\Gamma\left(\varepsilon_{p}\right) / 2 \pi}{\left(\varepsilon_{p}-\varepsilon_{\mathrm{res}}-\Delta\right)^{2}+\Gamma^{2 / 4}}\right\}^{1 / 2} .
$$

The details for obtaining $|\xi\rangle$ and $C\left(\varepsilon_{p}\right)$ can be found in Ref.?.

Our goal is to replace the continuum states $|p\rangle$ by the quasibound state $|\xi\rangle$ and furthermore to treat amplitudes with the index $\xi$ similarly as amplitudes specified by true bound states. In the

9 One may also use modified continuum wave functions |c $\rangle$ constructed orthogonal to $|\xi\rangle{ }^{10}$.

10 H. L. WANG and C. M. ShakrN, preprint (subm. to Phys. Letters). original problem we can distinguish three different particle-hole states:

$$
(v, \mu)=\{(r, i) ;(i, r)|(p, i) ;(i, p)|(c, i) ;(i, c)\} .
$$

Here $i$ characterizes hole states, $r$ true bound states and $c$ continuum states not belonging to the class defined by (II.3). In the new problem we want to deal with a new classification given by ${ }^{9}$

$$
(\boldsymbol{v}, \mu)=\{(r, i) ;(i, r)|(\xi, i) ;(i, \xi)|(c, i) ;(i, c)\} .
$$

In order to have a clear distinction we will label the particle-hole amplitudes, matrix elements etc. in the new classification by $\hat{\varrho}_{\lambda \sigma, M}, \hat{I}$ etc. Since in the second term of (II.1) only the wave functions within the range of the particle-hole force are needed we can insert there the quantities according to the new classification. We obtain then for the relevant term $((v \mu)$ according to (II.6)!)

$$
\begin{gathered}
\sum_{(\sigma \lambda) \in(p, i)} \tilde{I}_{\nu \sigma \mu \lambda} \hat{\varrho}_{\lambda \sigma, M} \approx \sum_{i} N_{\bar{\xi}}\left\{\hat{I}_{\nu i \mu \xi} \hat{\varrho}_{\xi i, M}\right. \\
\left.+\hat{I}_{\boldsymbol{\nu} \xi \mu i} \hat{\varrho}_{i \xi, M}\right\},
\end{gathered}
$$

with $^{11}$ :

$$
N^{\bar{\xi}}:=\int_{0}^{\varepsilon_{\max }} C^{2}\left(\varepsilon_{p}\right) \mathrm{d} \varepsilon_{p} \approx 1 .
$$

We have to be mindful of that Eq. (II.1) does not apply a priori to $\hat{\varrho}_{\xi \sigma, M}$ since $|\xi\rangle$ is not an eigenstate of the shell model hamiltonian. In the next section we are going to derive the equations for these quantities.

\section{RPA-Equations in the Resonant Approximation}

Using (II.7), (II.8) and (II.3) we can rewrite (II.1) as follows

$$
\begin{gathered}
\tilde{\varrho}_{\nu \mu, M}=\frac{\left(n_{\mu}-n_{\nu}\right)}{E_{M}+\varepsilon_{\mu}-\varepsilon_{v}-i \eta\left(n_{v}-n_{\mu}\right)}\left\{i \eta \delta_{k_{0} \nu} \delta_{j_{0} \mu} \delta_{M, S}\right. \\
\left.+2 \pi D_{v \mu} \sum_{\sigma \lambda} \hat{I}_{\nu \sigma \mu \lambda} \hat{\varrho}_{\lambda \sigma, M}\right\},
\end{gathered}
$$

with

$$
D_{v \mu}:= \begin{cases}1 & (v, \mu) \notin(p, i) ;(i, p), \\ C\left(\varepsilon_{v}\right) & v \in p, \\ C\left(\varepsilon_{\mu}\right) & \mu \in p .\end{cases}
$$

11 C. Mahaux and H. A. Weidenmüller, Shell-Model Approach to Nuclear Reactions, John Wiley, New York 1969 , p. 82 . 
Here, the quantity $\hat{I} \hat{\varrho}$ is labelled according to (II.6). We can distinguish three possibilities for $|\boldsymbol{M}\rangle$ :

a)

$$
|M\rangle=|B\rangle .
$$

From (II.3) we deduce the following relations:

$$
\tilde{\varrho}_{v \mu, B}=\left\{\begin{array}{l}
\hat{\varrho}_{v \mu, B} \quad(v \mu) \notin(p, i) ;(i, p), \\
C\left(\varepsilon_{v}\right) \hat{\varrho}_{\xi \mu, B} \quad v \in p, \\
C\left(\varepsilon_{\mu}\right) \varrho_{v \xi, B} \quad \mu \in p .
\end{array}\right.
$$

Insertion of (III.3) into (III.1) gives the following equation for $\hat{\varrho}_{v \mu, B}$ :

$\hat{\varrho}_{v \mu, B}=\left(n_{\mu}-n_{v}\right) \hat{f}_{\nu \mu}^{-1}\left(E_{B}\right) \sum_{\sigma \lambda} 2 \pi \hat{I}_{\nu \sigma \mu \lambda} \hat{\varrho}_{\lambda \sigma, B}$.

If either $v$ or $\mu$ is equal to $\xi$ this equation is obtained by multiplying (III.1) with $C\left(\varepsilon_{p}\right)$ and integration over the energy. Hence we have the following definitions for $\hat{f}_{\nu \mu}^{-1}(\Gamma=\eta \text { below threshold })^{11}$ :

$$
\begin{aligned}
& \hat{f}_{v \mu}^{-1}\left(E_{B}\right):=\left\{E_{B}+\varepsilon_{\mu}-\varepsilon_{v}-i \eta\left(n_{v}-n_{\mu}\right)\right\}^{-1} \\
& \text { for }(v, \mu) \neq(\xi, i) ;(i, \xi) \text {, } \\
& \hat{f}_{\xi \mu}^{-1}\left(E_{B}\right):=\int \frac{\mathrm{d} \varepsilon_{p}}{2 \pi}\left(\frac{\Gamma\left(\varepsilon_{p}\right)}{\left(\varepsilon_{p}-\varepsilon_{\mathrm{res}}-\Delta\right)^{2}+\Gamma^{2 / 4}}\right. \\
& \left.\cdot \frac{1}{E_{B}+\varepsilon_{\mu}-\varepsilon_{p}+i \eta}\right) \\
& \approx\left\{E_{B}+\varepsilon_{\mu}-\varepsilon_{\mathrm{res}}-\Delta+\frac{i}{2} \Gamma\left(E_{B}+\varepsilon_{\mu}\right)\right\}^{-1} . \\
& \hat{f}_{\nu \xi}^{-1}\left(E_{B}\right):=\int \frac{\mathrm{d} \varepsilon_{p}}{2 \pi}\left(\frac{\Gamma\left(\varepsilon_{p}\right)}{\left(\varepsilon_{p}-\varepsilon_{\mathrm{res}}-\Delta\right)^{2}+\Gamma^{2 / 4}}\right. \\
& \left.\cdot \frac{1}{E_{B}+\varepsilon_{p}-\varepsilon_{v}-i \eta}\right) \\
& \approx\left\{E_{B}+\varepsilon_{\mathrm{res}}+\Delta-\varepsilon_{v}-\frac{i}{2} \Gamma\left(E_{B}-\varepsilon_{v}\right)\right\}^{-1} .
\end{aligned}
$$

If approximation (II.8) is not valid one has to replace $\varrho$ in (III.4) by $N \hat{\varrho}$.

b)

$$
|\boldsymbol{M}\rangle=|S\rangle \equiv\left|S, k_{0} j_{0}\right\rangle \text { with } k_{0} \notin p .
$$

The equation for these amplitudes can be derived in the same manner as in the preceding subsection. We get:

$$
\begin{aligned}
\hat{\varrho} v \mu, S=\left(n_{\mu}-\right. & \left.n_{\nu}\right)\left\{\delta_{k_{0} \nu} \delta_{j_{0} \mu}\right. \\
& \left.+\hat{f}_{\nu \mu}^{-1}\left(E_{S}\right) \sum_{\sigma \lambda} 2 \pi \hat{I}_{\nu \sigma \mu \lambda} \hat{\varrho} \lambda \sigma, S\right\} .
\end{aligned}
$$

c) $\quad|M\rangle=|S\rangle \equiv\left|S, k_{0} j_{0}\right\rangle$ with $k_{0}=p_{0} \in p$.

The scattering state was defined by ${ }^{3}$

$$
|S\rangle=\left(z_{j_{0}} z_{p_{0}}\right)^{-1 / 2} \frac{i \eta}{E_{S}-H+i \eta} \psi_{p_{0}}^{\dagger} \psi_{j_{0}}|0\rangle,
$$

therefore it seems reasonable according to our assumptions to approximate the scattering state in the interior (localized state) by

with :

$$
\left|S_{\mathrm{L}}\right\rangle \approx C\left(\varepsilon_{p_{0}}\right)|\hat{S}\rangle
$$

$|\hat{S}\rangle:=\left(z_{j_{0}} z_{\xi_{0}}\right)^{-1 / 2} \frac{i \eta}{E_{S}-H+i \eta} \psi_{\xi_{0}}^{\dagger} \psi_{j_{0}}|0\rangle$.

Since $|\xi\rangle$ is concentrated in the interior it should be sufficient to treat $|\hat{S}\rangle$. If neither $v$ or $\mu$ belongs to $p$ one obtains,

$$
\hat{\varrho}_{v \mu, \hat{S}}=\left(n_{\mu}-n_{v}\right) \hat{f}_{v \mu}^{-1}\left(E_{S}\right) \sum_{\sigma \lambda} 2 \pi \hat{I}_{\nu \sigma \mu \lambda} \hat{\varrho}_{\lambda \sigma, \hat{S}}
$$

If - for instance $-v$ belongs to $p$ we get from Eq. (III.1):

$$
\begin{aligned}
\hat{\varrho}_{p i, S}= & \left(n_{i}-n_{p}\right)\left\{\delta_{i j_{0}}\left\langle p \mid p_{0}\right\rangle\right. \\
& \left.+\frac{C\left(\varepsilon_{p}\right) C\left(\varepsilon_{p 0}\right)}{E_{S}+\varepsilon_{i}-\varepsilon_{p}+i \eta} \sum_{\sigma \lambda} \hat{I}_{\xi \sigma i \lambda} \hat{\varrho}_{\lambda \sigma, \hat{S}}\right\}
\end{aligned}
$$

In the interior now the following replacement holds:

$$
\hat{\varrho}_{p i, S} \approx C\left(\varepsilon_{p}\right) \hat{\varrho}_{\xi i, S}=C\left(\varepsilon_{p}\right) C\left(\varepsilon_{p 0}\right) \hat{\varrho}_{\xi i, \hat{S}} .
$$

Furthermore, since we are only interested in the interior region we can insert instead of $\left\langle p \mid p_{0}\right\rangle$ the following expression:

$$
\left\langle p \mid p_{0}\right\rangle_{\mathrm{L}} \approx C\left(\varepsilon_{p}\right) C\left(\varepsilon_{p_{0}}\right) \delta_{\xi \xi_{0}} .
$$

The quantum number set $\xi\left(\xi_{0}\right)$ coincides with the quantum number set $p\left(p_{0}\right)$ if we exclude the energy $(\langle\xi \mid \xi\rangle=1)$. Use of (III.14) and (III.15) leads to:

$\hat{\varrho}_{\xi i, \hat{S}}=\delta_{i j_{0}} \delta_{\xi \xi_{0}}+\hat{f}_{\xi i}^{-1}\left(E_{S}\right) \sum_{\sigma \lambda} 2 \pi \hat{I}_{\xi \sigma i \lambda} \hat{\varrho}_{\lambda \sigma, \hat{S}}$.

Analogously we get:

$$
\hat{\varrho}_{i \xi, \hat{S}}=-2 \pi \hat{f}_{i \xi}^{-1}\left(E_{S}\right) \sum_{\sigma \lambda} 2 \pi \hat{I}_{i \sigma \xi \lambda} \hat{\varrho}_{\lambda \sigma, \hat{S}} .
$$

Equations (III.4), (III.8), (III.12), (III.16) and (III.17) provide the system of equations necessary for the RPA-treatment with inclusion of the continuum. The transformation to the original problem is formally given by Eq. (III.1).

In the next section we are going to derive a model in which all matrix elements containing single-particle states $|c\rangle$ are approximated by a separable interaction.

\section{Model Equations}

We can split off the sum over quantum numbers in our equations in two terms, where the first sum contains only the summation over bound or quasi- 
bound states. In the second sum we replace the matrix elements by separable ones i.e.:

$$
2 \pi \hat{I}_{\nu \sigma \mu \alpha}=\lambda \hat{W}_{\nu \mu} \hat{W}_{\sigma \alpha},
$$

so obtaining:

$$
\begin{aligned}
2 \pi \sum_{\sigma \alpha} \hat{I}_{\nu \sigma \mu \alpha} \hat{\varrho} \alpha \sigma, M= & 2 \pi \sum_{\sigma \alpha}^{\prime} \hat{I}_{\nu \sigma \mu \alpha} \varrho \alpha \sigma, M \\
& +\lambda \hat{W}_{\nu \mu} \sum_{\sigma \alpha}^{\prime \prime} \hat{W}_{\sigma \alpha} \hat{\varrho}_{\alpha \sigma, M},
\end{aligned}
$$

where the restricted sums are defined as follows
$\left(U_{v \mu}\right.$ is an arbitrary function of $\nu$ and $\left.\mu\right)$ :

$$
\begin{aligned}
& \sum_{\nu \mu}^{\prime} \hat{U}_{v \mu}=\sum_{r i}\left(\hat{U}_{\mathrm{r} i}+\hat{U}_{i \mathrm{r}}\right)+\sum_{i \xi_{p}}\left(\hat{U}_{\xi i}+\hat{U}_{i \xi}\right), \\
& \sum_{\nu \mu}^{\prime \prime} \hat{U}_{v \mu}=\sum_{c i}\left(\hat{U}_{c i}+\hat{U}_{c i}\right) .
\end{aligned}
$$

Insertion of (IV.2) into Eq. (III.4) leads to the following eigenvalue problem:

$$
\begin{array}{r}
\sum_{\lambda \sigma}^{\prime}\left\{\hat{A}_{v \sigma \mu \lambda}+\hat{C}_{\nu \sigma \mu \lambda}^{B}-\delta_{v \lambda} \delta_{\sigma \mu} E_{B}\right\} \hat{\varrho}_{\lambda \sigma, B}=0 \\
\text { for } v \mu \notin(c i) ; \quad(i c),
\end{array}
$$

where we have introduced the following abbreviations:

$$
\begin{array}{cl}
U_{B}:=\lambda \sum_{\nu \mu}^{\prime \prime}\left|\hat{W}_{\nu \mu}\right|^{2}\left(n_{\mu}-n_{\nu}\right) \frac{1}{E_{B}+\varepsilon_{\mu}-\varepsilon_{\nu}-i \eta\left(n_{\nu}-n_{\mu}\right)}, \\
m_{B}:=U_{B} \sum_{\alpha \beta}^{\prime}\left(n_{\alpha}-n_{\beta}\right)\left|\hat{W}_{\beta \alpha}\right|^{2}, \quad(\text { IV.7) } & \text { with } \quad D_{B}:=\sum_{\sigma \lambda}^{\prime} \hat{W}_{\sigma \lambda} \hat{\varrho}_{\lambda \sigma, B} .
\end{array}
$$

$\hat{A_{\nu \sigma \mu \lambda}}:=\left(n_{\mu}-n_{v}\right) 2 \pi \hat{I_{\nu \sigma \mu \lambda}}-\delta_{\lambda v} \delta_{\sigma \mu}\left(\hat{f_{v \mu}}\left(E_{B}\right)-E_{B}\right)$,

$$
\hat{C}_{v \sigma \mu \lambda}^{B}:=\left(n_{\mu}-n_{\nu}\right) \lambda \hat{W}_{\nu \mu} \frac{U_{B} \sum_{\alpha \beta}^{\prime} \hat{W}_{\alpha \beta} \hat{A}_{\alpha \sigma \beta \lambda}}{E_{B}\left(1-U_{B}\right)-\lambda m_{B}}
$$

The amplitudes with one index belonging to the continuum are given by:

$\hat{\varrho}_{v \mu, B}=\frac{\left(n_{\mu}-n_{\nu}\right)}{E_{B}+\varepsilon_{\mu}-\varepsilon_{\nu}-i \eta\left(n_{\nu}-n_{\mu}\right)} \lambda \hat{W}_{\nu \mu} D_{B} \frac{1}{1-U_{B}}$,

Equation (IV.5) provides a matrix equation which may be solved by iteration. For $\Gamma \rightarrow 0$ and $\lambda \rightarrow 0$ we get the standard nuclear structure problem which might be used as the starting of the iteration procedure. Above threshold one will obtain complex energy eigenvalues due to presence of $U_{B}$ (even if $\Gamma \rightarrow 0$ ). Since we have removed the shape resonances it should be easy in a practical calculation to interpolate $U_{B}$ as a function of $E$. When one has solved the eigenvalue problem (IV.5) the amplitudes containing continuum single-particle states $|c\rangle$ can be obtained from (IV.10).

The subcase b) can be treated in a similar manner. Due to the inhomogenous term in Eq. (III.8) we get the following inhomogenous matrix equation:

$$
\sum_{\sigma \lambda}^{\prime}\left\{\hat{A}_{v \sigma \mu \lambda}+\hat{C}_{\nu \sigma \mu \lambda}^{S}-\delta_{v \lambda} \delta_{\sigma \mu} E_{S}\right\} \hat{\varrho}_{\lambda \sigma, S}=\hat{d}_{v \mu}^{k_{0} j_{0}, S} \text { for }(v \mu) \notin(c, i) ;(i, c) .
$$

with:

$$
\hat{d}_{\nu \mu}^{k_{0} j_{0}, S}:=-\lambda\left(n_{\mu}-n_{v}\right)\left(n_{j_{0}}-n_{k_{0}}\right) \hat{W}_{\nu \mu} \hat{W}_{k_{0} j_{0}} \frac{E_{S}}{E_{S}\left(1-U_{S}\right)-\lambda m_{S}} .
$$

The amplitudes with one continuous index are given by:

$$
\hat{\varrho}_{v \mu, S}=\left(n_{\mu}-n_{v}\right)\left\{\delta_{k_{0} v} \delta_{j_{0} \mu}+\frac{\lambda \hat{W}_{v \mu}}{E_{S}+\varepsilon_{\mu}-\varepsilon_{\nu}-i \eta\left(n_{v}-n_{\mu}\right)} \frac{1}{1-U_{S}}\left[D_{S}+\hat{W}_{k_{0} j_{0}}\right]\right\} \text { for } v \mu \in(c, i) ;(i, c) \text {. }
$$

Since $d$ is non zero one has in this case only to invert a matrix of finite dimension to obtain the solution. As expected these solutions have resonances for $E_{S}=\operatorname{Re} E_{\mathrm{B}}$.

If the incoming particle belongs to the class $p$ the inhomogenous term occurs in the Eq. (III.16) for $\hat{\varrho}_{\xi i, \hat{S}}$ and not in $\hat{\varrho}_{c i, \hat{S}}$ as in subcase $b$. For this reason we get a different inhomogenous term in comparison with (IV.12). One obtains:

$$
\sum_{\nu \lambda}^{\prime}\left\{\hat{A}_{v \sigma \mu \lambda}^{\hat{S}}+\hat{C}_{v \sigma \mu \lambda}^{\hat{S}}-\delta_{\nu \lambda} \delta_{\sigma \mu} E_{S}\right\} \hat{\varrho} \lambda \lambda \sigma, \hat{S}=\hat{d}_{\nu \mu}^{\xi_{v} j_{0}, \hat{S}} \quad \text { for } \nu \mu \notin(c, i) ;(i, c) .
$$


with

$$
\hat{d}_{\nu \mu}^{\xi_{0} j_{0}, \hat{S}}:=\left(n_{\nu}-n_{\mu}\right){\hat{f_{\xi_{0}} j_{0}}}_{\left.E_{S}\right)} \frac{1}{1-U \hat{S}}\left\{\delta_{j_{0} \mu} \delta_{\nu \xi_{0}}+\frac{\lambda U \hat{\mathrm{S}} \hat{W}_{v \mu} \hat{W}_{\xi_{0} j_{0}}}{E_{S}(1-U \hat{S})-\lambda m_{\hat{S}}}\right\} .
$$

The equivalent relation to (IV.14) turns out to be:

$$
\hat{\varrho}_{v \mu, \hat{S}}=\frac{n_{\mu}-n_{\nu}}{E_{S}+\varepsilon_{\mu}-\varepsilon_{v}-i \eta\left(n_{\nu}-n_{\mu}\right)} \lambda \hat{W}_{v \mu} \frac{D \hat{S}}{1-U \hat{S}} \text { for }(v \mu) \in(c i) ;(i c) .
$$

Both solutions have as in the former case resonances for $E_{S} \rightarrow \operatorname{Re} E_{B}$.

The final solution according to the classification (II.5) can be obtained for the bound states $|B\rangle$ with the help of the relations (III.3). In the scattering problem we get approximately for the case $k_{0} \notin p$ :

$$
\tilde{\varrho}_{v \mu, S}= \begin{cases}\hat{\varrho}_{v \mu, S} & (v \mu) \in(r, i) ;(i, r), \\ C\left(\varepsilon_{v}\right) \hat{\varrho}_{\xi \mu, S} & v \in p, \\ C\left(\varepsilon_{\mu}\right) \hat{\varrho}_{v \xi, S} \quad \mu \in p, & \left(n_{\mu}-n_{v}\right) \\ \delta_{v k_{0}} \delta_{\mu j_{0}}+\frac{E_{S}+\varepsilon_{\mu}-\varepsilon_{v}-i \eta\left(n_{v}-n_{\mu}\right)}{E} \lambda W_{v \mu} \frac{1}{1-U_{S}}\left[D_{S}+W_{k_{0} j_{0}}\right] \quad(v \mu) \in(c i) ;(i c) .\end{cases}
$$

If $k_{0}=p_{0} \in p$ one can derive the following relations using (III.1), (III.10), (III.12), (III.16) and (III.17):

$$
\hat{\varrho}_{v \mu, S}= \begin{cases}\frac{n_{\mu}-n_{v}}{E_{S}+\varepsilon_{\mu}-\varepsilon_{v}-i \eta\left(n_{v}-n_{\mu}\right)} \frac{\lambda W_{v \mu} D \hat{S} C\left(\varepsilon_{p_{0}}\right)}{1-U \hat{S}} & (v \mu) \in(c, i) ;(i, c), \\ C\left(\varepsilon_{p_{0}}\right) \hat{\varrho}_{v \mu, \hat{S}} & (v \mu) \in(r, i) ;(i, r), \\ C\left(\varepsilon_{p_{0}}\right) C\left(\varepsilon_{p}\right) \hat{\varrho}_{i \xi, \hat{S}} & v=i, \mu=p, \\ \delta_{p p_{0}} \delta_{i j_{0}}+\frac{C\left(\varepsilon_{p}\right)_{0} C\left(\varepsilon_{p}\right)}{\left\lfloor E_{S}+\varepsilon_{i}-\varepsilon_{p}+i \eta\right.}\left[\hat{f}_{\xi i}\left(E_{S}\right) \hat{\varrho}_{\xi i, \hat{S}}-\frac{\hat{f}_{\xi_{0}} j_{0}\left(E_{S}\right)}{1-U \hat{S}} \delta_{j_{0} i} \delta_{\xi \xi_{0}}\right] & v=p, \quad \mu=i .\end{cases}
$$

The second and third relations in (IV.18) and the third relation in (IV.19) are valid only in the interior.

From (IV.18) and (IV.19) the wanted expressions for the $S$-matrix and the $T$-matrix can now be read off. After reinserting the single-particle phase factors taken out by using standing wave boundary conditions, one obtains:

with

$$
S_{k i, k_{0} j_{0}}(E)=\exp \left\{2 i \delta_{k_{0}}\right\} \delta_{k k_{0}} \delta_{i j_{0}}-2 \pi i \delta\left(E_{k_{0} j_{0}}-E\right) T_{k i, k_{0} j_{0}}(E),
$$

$$
\begin{aligned}
& T_{k i, k_{0} j_{0}}(E):=\lambda W_{k i} \frac{1}{1-U_{S}}\left[D_{S}+W_{k_{0} j_{0}}\right] \exp \left\{i\left(\delta_{k_{0}}+\delta_{k}\right)\right\} \text { for } k \notin p, k_{0} \notin p, \\
& T_{p i, k_{0} j_{0}}(E):=C\left(\varepsilon_{p}\right) \hat{\varrho}_{\xi i, S} \exp \left\{i\left(\delta_{k_{0}}+\delta_{p}\right)\right\} \hat{f}_{\xi i(E)} \text { for } k=p, k_{0} \notin p, \\
& T_{k i, p_{0} j_{0}}(E):=\lambda W_{k i} \frac{1}{1-U_{\hat{S}}} D_{\hat{S}} C\left(\varepsilon_{p_{0}}\right) \exp \left\{i\left(\delta_{k}+\delta_{p_{0}}\right)\right\} \text { for } k \notin p, k_{0}=p_{0} \in p, \\
& T_{p i, p_{0} j_{0}}(E):=C\left(\varepsilon_{p_{0}}\right) C\left(\varepsilon_{p}\right)\left[\hat{f}_{\xi i}(E) \hat{\varrho}_{\xi i, \hat{S}}-\frac{\delta_{j_{0} i} \delta_{\xi \xi_{0}}}{1-U_{\hat{S}}} \hat{f}_{\xi_{0} j_{0}}(E)\right] \exp \left\{i\left(\delta_{p_{0}}+\delta_{p}\right)\right\} \\
& \text { for } k=p, k_{0}=p_{0} \in p .
\end{aligned}
$$

For $E_{S}=\varepsilon_{p}-\varepsilon_{i}$ and $\varepsilon_{p} \rightarrow \varepsilon_{\text {res }}+\Delta$ the $S$-matrix does not possess-as expected-single-particle resonance, since (IV.20) takes the following form

$$
e^{i 2 \delta}\left\{1+2 \pi i \frac{\Gamma / 2 \pi\left[\left(\varepsilon_{p}-\varepsilon_{\mathrm{res}}-\Delta\right)+i / 2 \Gamma\right]}{\left(\varepsilon_{p}-\varepsilon_{\mathrm{res}}-\Delta\right)^{2}+\Gamma^{2 / 4}}(1+\ldots)\right\} \approx 1,
$$

where we have supressed the $\delta$-function of energy.

\section{Summary}

A model for calculating the nucleon-nucleus scattering on one-hole type nuclei has been settled down in the framework of the renormalized RPA- theory, in which the shape resonant shell model states have been treated approximately as bound states. With a separable ansatz for the remaining continuum matrix-elements we have been able to derive a system of equations, which can be solved 
up to integrations over known nonresonant functions by standard matrix methods. ${ }^{12}$

12 It was shown recently that one can also use Weinberg's method for the treatment of the continuum-continuum interaction in the unrenormalized RPA-scattering problem ${ }^{13}$.

13 J. N. Ginocchio, T. H. Schucan, and H. A. WeidenMüller, Phys. Rev. C 1, 55 [1970].
Acknowledgments

The author would like to thank Dr. L. GARSIDE for helpful discussions and wishes to express his gratitude to the members of the theoretical nuclear physics group in Berkeley for their hospitality. The Deutsche Forschungsgemeinschaft, Germany, supported this work by a grant.

\title{
Low-Energy Electron Diffraction from $\mathrm{Ag}(111)$ : Intensity-versus-Energy Curves and Absolute Intensity of the (00) Beam*
}

\author{
Max G. Lagally \\ Fritz-Haber-Institut der Max-Planck-Gesellschaft, Berlin-Dahlem, Germany **, *** \\ (Z. Naturforsch. 25 a, 1567-1578 [1970] ; received 17 August 1970)
}

\begin{abstract}
The intensity of the (00) beam of a (111) surface of $\mathrm{Ag}$ has been measured with a Faraday cage as a function of the energy of the incident beam $(10<E<280 \mathrm{eV})$, the grazing angle of incidence $\left(46.5^{\circ}<\varphi<83.5^{\circ}\right)$, two azimuths differing by $180^{\circ}$, and the temperature. The $I$ vs $E$ curves, when compared with data for $\mathrm{Ag}(111)$ of other workers who have used different methods of surface preparation, show good agreement in the structure over the whole range of incident angles, indicating that LEED is not strongly sensitive to surface condition. The $I$ vs $E$ curves for the two azimuths are identical, a necessary result of the reciprocity theorem. For comparison with the $I$ vs $E$ structure, a complete 3-beam geometric model is used. This differs from a simple Ewald construction in that it considers also the Bragg conditions between intermediate beams and the final beam. It also requires that there be no difference in the effect of intermediate forward and backward scattered beams. It is shown that the number of possible beams is much too large even at low energies to make positive identification of any structure in the $I$ vs $E$ curves. A comparison with a rigorous multiple-scattering theory yields agreement in the number and position of peaks, but not in heights and widths of peaks. The possibility of comparison of absolute intensities in theory and experiment is investigated and an attempt is made to remove the major differences. Intensity vs temperature measurements are made at closely spaced energies in order to extract the rigid-lattice scattering. Correction of this intensity for surface plasma losses leads finally to maximum scattered intensities of $2 \%$ at $100 \mathrm{eV}, 10 \%$ at $60 \mathrm{eV}$, and up to $40 \%$ at energies below $20 \mathrm{eV}$.
\end{abstract}

Although much of the experimental work in lowenergy electron diffraction has dealt with the effect on the diffraction of some change in the surface or its condition, such as adsorption or reconstruction, lately there have appeared a number of papers dealing with the diffraction process itself at simple crystal surfaces. The data are generally presented in the form of intensity versus energy $(I$ vs $E$ ) curves for particular beams ${ }^{1-3}$ or sometimes as rocking curves $^{4}$ or RENNINGER (rotation) plots ${ }^{4-6}$. Understanding these intensities of the diffraction of slow electrons is the goal of the main theoretical effort.

Reprints request to Fritz-Haber-Institut der Max-PlanckGesellschaft, D-1000 Berlin 33, Faradayweg 4-6.

* Work performed under a stipend of the Max-Planck-Gesellschaft.

** Abt. Prof. Dr. K. Molière.

** Present address: Physics Dept., Univ. of Wisconsin, Madison, Wisconsin. Partial support by the U.S. Air Force Office of Scientific Research, Grant No. AF-AFOSR 69-1677.
This effort can be roughly divided into two groups; rigorous theories and simpler phenomenological models.

Of the simple models ${ }^{2-4}$, all have dealt only with the structure observed in the $I$ vs $E$ curves, and have made no attempt to deal with the magnitude of the intensities. They are based on the geometry of the diffraction in either a multiple-beam or bandstructure picture. Rigorous theories, on the other hand, calculate the diffracted intensity resulting from an infinite plane wave falling on a perfect, laterally semi-infinite model crystal using methods

1 C. D. Gelatt, JR., M. G. Lagally, and M. B. Webb, Bull. Amer. Phys. Soc. 15, 632 [1970].

2 M. P. Seah, Surface Sci. 17, 181 [1969].

3 L. G. Feinstein, Surface Sci. 19, 366 [1970].

4 A. Gervais, R. M. Stern, and M. Menes, Acta Cryst. A 24, 191 [1968].

5 M. B. Webb, Tran C. Ngoc, and M. G. Lagally, Bull. Amer. Phys. Soc. 15, 632 [1970].

6 M. G. Lagally and M. B. WeBb, to be published. 